\title{
II Centro di Riferimento per la Tipizzazione di Salmonella spp in Emilia-Romagna
}

\author{
Giuseppe Cirillo, Francesco Ortali \\ Eccellenza Regionale Biologia e Microbiologia S\&R ARPA Forli-Cesena
}

Key words: the genus Salmonella, D.R.C. (Distrectual Reference Centre),serotype identification

The Distrectual Reference Centre of Salmonella sp in Emilia-Romagna

\section{SUMMARY}

The steady actuality of genus Salmonella follows step by step the new Italian's eat habit (canteen, fast food etc...), this fact brought up a fast spreading of the bacteria inside the all of the Emilia Romagna food industry.

The need to set up Distrectual Reference Centres working inside a net system came about because of technical reasons (new methods, quality management, etc...) as well as economic ones (structures, staff, etc...). Activities of the D.R.C. (Distrectual Reference Centre) from 1997 till now are described.

The classical analytic protocol to isolate the Salmonella spp require enrichment steps of the sample (when necessary) biochemical identification (by dedicated systems) and a complete serological identification (serotyping). The Centre process samples coming from: human, food and environmental matrixes. It utilizes acceptation procedures and analytical methods Sinal approved. Data are sent to ISS in Rome every month. This procedure is part of the European Project ENTER-NET.

\section{RIASSUNTO}

La costante attualità del genus Salmonella segue di pari passo le nuove abitudini alimentari degli italiani (ristorazione collettiva, fast food etc.) che hanno portato ad una rapida proliferazione del settore agroalimentare in tutta la Regione Emilia Romagna.

L'esigenza di costituire Centri di Riferimento (Eccellenze) nell'ambito di un sistema nodale, trova le sue motivazioni da motivi tecnici (nuove metodologie, qualità, etc.) ed economici (strutture, personale, etc.). Si descrive l'attività, dal 1997, ad oggi del C.R.R.(Centro Regionale di Riferimento) per l'Emilia-Romagna. Le metodiche analitiche classiche per l'isolamento di Salmonella spp prevedono le fasi di arrichimento (del campione laddove necessita), l'isolamento in terreno selettivo, la tipizzazione biochimica (con sistemi dedicati) e l'identificazione completa del sierotipo (sierotipizzazione). II Centro processa campioni (ceppi) provenienti da matrici umane, alimentari e ambientali utilizzando procedure di accettazione e metodiche analitiche accreditate Sinal. I dati sono inviati mensilmente all'ISS di Roma nell'ambito del Progetto Europeo ENTER-NET.

\section{INTRODUZIONE}

Il genus Salmonella, è agente eziologico di una patologia prevalentemente gastroenterica. La sua costante attualità segue di pari passo le nuove abitudini alimentari degli italiani e i nuovi costumi (ristorazione collettiva, fast food etc...) che hanno portato ad una rapida proliferazione di industrie alimentari sparse un po' in tutta la nostra Regione (suinicole e lattiero casearie a Modena, Reggio Emilia e Parma, avicole e suinicole a Forlì e Cesena).

L'esigenza di costituire Centri di Riferimento (Eccellenze) nell'ambito di un sistema nodale, sorge da motivi culturali ed economici.

L'aumento della conoscenza, l'esigenza di specializzazione e le procedure della Qualità hanno portato, negli ultimi anni, all'impossibilità per ogni singolo Laboratorio di eseguire in loco ogni tipo- logia di prestazione analitica pena ripercussioni economiche su reagenti, personale, strutture, etc... La conoscenza microbiologica ha, d'altra parte, seguito lo sviluppo avutosi nelle altre discipline, raggiungendo oggi i suoi massimi livelli. Questo comporta un maggior onere, non sempre sostenibile, da parte di ogni singolo Laboratorio per essere in condizioni di rispondere alle richieste di prestazioni. La Microbiologia applicata, strettamente collegata alla ricerca pura, è passata nell'ultimo mezzo secolo da metodologie relativamente semplici che fornivano diagnosi indicative, a metodiche sempre più sofisticate che hanno portato all'identificazione completa e attendibile dei microorganismi. Le tappe della ricerca si sono avvalse della Microscopia, della Biochimica, dell'Immunologia e della Genetica. Questi presupposti giustificano i Centri di Eccellenza a cui i 
Laboratori periferici provinciali ARPA ER (clienti interni), i Laboratori Ospedalieri di Microbiologia e i Laboratori Microbiologici Privati (clienti esterni) afferiscono per il completamento di ricerche che sarebbe antieconomico e dispersivo condurre in sede.

L'attività del Centro di Riferimento Regionale è iniziata nel 1997, quando in seno all'ARPA Emilia Romagna si andava delineando quella che sarebbe stata la muova struttura che prevedeva l'accentramento di prestazioni analitiche specialistiche in centri regionali. $\mathrm{Fu}$ indicato Forlì in quanto nel suo Laboratorio di Microbiologia si effettuavano ancora ricerche sull'uomo (prevenzione malattie infettive) ed era esso stesso dotato di strumentazione automatica d'avanguardia oltre ad essere già da tempo referente dell'allora Progetto Salmnet.

\section{MATERIALI E METODI}

La ricerca di Salmonella spp da qualsiasi matrice si avvale di alcune fasi esecutive che prevedono un arricchimento del campione in apposito brodo, un suo isolamento e, dalle caratteristiche morfologiche delle colonie, una tipizzazione biochimica che identifica Salmonella spp.

L'identificazione finale e completa del sierotipo si ottiene tramite test sierologici. Ulteriori ricerche (lisotipia, tipizzazione fagica etc...) vengono effettuate, solo in casi dubbi, nelle tossinfezioni alimentari laddove è utile definire se effettivamente il microorganismo isolato dagli ammalati corrisponde a quello isolato nell'alimento incriminato (sede legale). Si applicano limitatamente a S. enteritidis e S. tiphymurium e non ha senso una ricerca routinaria se non inserita in un apposito progetto. L'identificazione sierologica costituisce la risultante (formula antigenica) delle risposte che il ceppo batterico fornisce quando è testato con antigeni somatici $(\mathrm{O})$ e ciliari $(\mathrm{H})$. Questa risultante è definita Sierotipo. La tabella di Kaupfmann White presenta circa 1800 sierotipi conosciuti del genere Salmonella che seguono distribuzioni geografiche particolari. Si evince che una ricerca completa risulta alquanto lunga e indaginosa. Antieconomico è poi lo stoccaggio degli antisieri, soggetti a scadenze (circa un anno) e non sempre completamente utilizzati. Se da una parte è importante che un Laboratorio di Microbiologia applichi procedure che prevedono l'identificazione biochimica di una specie microbica il prosieguo e il completamento della ricerca (analisi sierologica) è legato anche a fattori economici e di personale. Nasce di qui l'esigenza di Centri di raccolta e di riferimento. Esempi di questo tipo ne esistono: basta citare l'Istituto Pasteur di Parigi, ma, avvicinandoci, abbiamo l'e- sempio regionale attuatosi con il patrocinio di Società scientifiche (AMCLI, Società Italiana di Microbiologia) che hanno istituito centri di riferimento limitati a determinate specie batteriche (es. Micobatteri, Listeria, Legionella etc...) e siti presso Laboratori Ospedalieri o Policlinici universitari. Scopo di questi Centri è di fungere da supporto agli altri Laboratori nel completamento di ricerche che singolarmente avrebbero comportato spese non giustificabili, oltrechè elaborare i dati da un punto di vista epidemiologico.

Al Laboratorio Periferico resta, a tipizzazione biochimica effettuata, la preparazione del ceppo, la compilazione della scheda Enternet e l'invio al Laboratorio Regionale di Riferimento. L'inoltro al Centro di Riferimento dei ceppi batterici avviene seguendo Procedure esecutive dedicate.

\section{RISULTATI E DISCUSSIONE}

L'attività del Centro di Riferimento dalla sua fondazione è sintetizzata dalla tabella 1 in cui sono indicati gli isolamenti complessivi annuali per Provincia. Si può notare come, nell'arco degli anni l'attività si è progressivamente estesa a tutte le Province. Dobbiamo però segnalare che, i dati di derivazione ambientali sono pressoché completi e molto vicini alla realtà, provenendo da tutti i Laboratori di Microbiologia delle nove Sezioni ARPA. Diverso è il discorso per i dati umani.

Come illustrato nella tabella 2), ove sono esposti i dati 2005, la netta differenza quantitativa fra isolamenti ambientali e alimentari, è dovuta al fatto che questi ultimi sono passati completamente di pertinenza dei Laboratori Provinciali di Zooprofilassi, restando all'ARPA solo gli alimenti di origine non-animale. Gli isolamenti umani, poi, sono altamente sottostimati in quanto al circuito Enternet tuttora mancano i Grandi Policlinici della nostra Regione come Bologna, Modena e Parma. Si può affermare senza tema di errore che gli isolamenti umani reali si aggirano attorno a un valore superiore al doppio di quanto citato nella tabella 2 .

Nella tabella 3 sono elencati in modo completo tutti i sierotipi isolati nel 2005 suddivisi per matrice e per Gruppi d'appartenenza: si nota che i gruppi maggiormente rappresentati sono il $\mathrm{B}, \mathrm{C}$, D, E, F.

La tabella 4 mostra, in confronto, i primi dieci sierotipi isolati da matrici ambientali e umane. $\mathrm{Si}$ nota che la corrispondenza esiste solo per 4 specie (SS. typhimutium, infantis, london e montevideo). Inoltre nell'ambiente esiste una marcata eterogeneità di specie, infatti le prime dieci specie vanno a costituire il $66 \%$ delle totali specie isolate (900) contro il 95\% delle prime dieci di origine umana su 364 totali. Una certa attenzione va fatta 
su $S$. veneziana che viene isolata costantemente da anni da matrici ambientali senza avere un dovuto riscontro negli alimenti o nell'uomo. L'isolamento di questo sierotipo si verifica costantemente anche nelle altre Regioni. Non esistono tutt'oggi motivi che possano creare allerta nella sanità pubblica, ma non bisogna dimenticare che già in passato sierotipi non endemici come S. wien (1974) e S. jerusalem (1985) sono stati causa di episodi epidemici per poi tornare nell'anonimato.

Tabella I. Totale isolamenti annuali 1997/2005

\begin{tabular}{|c|c|c|c|c|c|c|c|c|c|c|}
\hline & ANNO & 1997 & 1998 & 1999 & 2000 & 2001 & 2002 & 2003 & 2004 & 2005 \\
\hline I & Piacenza & & & 14 & 79 & 135 & 102 & 80 & 121 & 135 \\
\hline 2 & Parma & & & 134 & 186 & 156 & 52 & 75 & 57 & 49 \\
\hline 3 & Reggio Emilia & & & & & 385 & 380 & 474 & 346 & 458 \\
\hline 4 & Modena & & & & 278 & 273 & 158 & 145 & 179 & 11 \\
\hline 5 & Bologna & & & 4 & & 9 & 5 & 3 & 17 & 21 \\
\hline 6 & Ferrara & & & 3 & & 165 & 148 & 124 & 82 & 42 \\
\hline 7 & Forlì & 274 & 285 & 321 & 199 & 234 & 193 & 141 & 250 & 227 \\
\hline 8 & Ravenna & & 78 & 126 & 111 & 64 & 75 & 169 & 187 & 291 \\
\hline 9 & Rimini & & & & 18 & 8 & 56 & 53 & 139 & 88 \\
\hline & Totale & 274 & 363 & 632 & 871 & 1429 & 1169 & I 264 & 1378 & 1322 \\
\hline
\end{tabular}

Tabella 2. Anno 2005: distribuzione degli isolamenti per matrice e Provincia

\begin{tabular}{|c|c|c|c|c|c|c|c|c|}
\hline 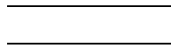 & ACQ.SUP. & ACQ.SCA & FANGHI & ALIMENTI & CQ & UOMO & ALTRO & TOTALE \\
\hline PIACENZA & 135 & - & - & - & - & - & - & 135 \\
\hline PARMA & 49 & - & - & - & - & - & - & 49 \\
\hline REGGIO E. & 277 & 35 & 10 & 8 & 9 & 118 & 3 & 458 \\
\hline MODENA & - & 11 & - & - & - & - & - & II \\
\hline BOLOGNA & - & - & - & 20 & - & - & 1 & 21 \\
\hline FERRARA & - & - & - & - & - & 42 & - & 42 \\
\hline RAVENNA & 169 & 22 & 5 & 2 & 4 & - & 2 & 227 \\
\hline FORLI & 89 & 1 & $\mathrm{I}$ & 6 & 19 & 176 & - & 291 \\
\hline RIMINI & 85 & - & 3 & - & - & - & - & 88 \\
\hline TOTALE & 810 & 69 & 19 & 36 & 32 & 336 & 6 & 1322 \\
\hline
\end{tabular}

Legenda: ACQ.SUP. acque superficiali, ACQ.SCA. acque di scarico, FANGHI fanghi, ALIMENTI alimentari, CQ controllo di qualità, UOMO umani.

Tabella 3. Distribuzione dei sierotipi isolati nel 2005 per matrice di derivazione

\begin{tabular}{|c|c|c|c|c|c|c|}
\hline GRUPPO & SIEROTIPO & AMBI & ALIM & UOMO & C. $\mathbf{Q}$. & TOTALE \\
\hline \multirow[t]{2}{*}{ A O: I,2 } & Nitra & 25 & & & & 25 \\
\hline & Koessen & 7 & & & & 7 \\
\hline \multirow[t]{9}{*}{ B O:4,5 } & Typhimurium & $|5|$ & 9 & 205 & & 365 \\
\hline & Sant Paul & 5 & & & & 5 \\
\hline & Derby & 38 & 2 & & & 40 \\
\hline & Agona & 38 & & & & 38 \\
\hline & Bredeney & 9 & & 1 & & 10 \\
\hline & Branderburg & 9 & & & & 9 \\
\hline & Heidelberg & 3 & & & & 3 \\
\hline & Mons & & $\mathrm{I}$ & & & $\mathrm{I}$ \\
\hline & Haifa & 6 & & & & 6 \\
\hline GRUPPO & SIEROTIPO & AMBI & ALIM & UOMO & C. $\mathbf{Q}$. & TOTALE \\
\hline \multirow[t]{10}{*}{ CI O:6,7 } & Ohio & 2 & & & & 2 \\
\hline & Livingstone & 24 & & 3 & & 27 \\
\hline & Infantis & 56 & $\mathrm{I}$ & 24 & & 81 \\
\hline & Virchow & 13 & & & & 13 \\
\hline & Montevideo & 34 & & 4 & & 38 \\
\hline & Braenderup & 8 & 5 & 4 & & 17 \\
\hline & Galiema & 7 & & & & 7 \\
\hline & Irumu & I & & & & I \\
\hline & Jerusalem & 6 & & & & 6 \\
\hline & Thompson & 2 & & & & 2 \\
\hline
\end{tabular}


Tabella 3. Segue GRUPPO

SIEROTIPO

Muenchen

Manhattan

Kottbus

Newport

Glostrup

B.Morbificans

Loanda

Blockley

Hadar

Tshiongwe

Chincol

Litchfield

Jerusalem

\section{AMBI}

18

18

7

II

4

I

7

7

I

I

2

\section{ALIM}

UOMO

C. $\mathbf{Q}$.

7

I

6

12

\section{TOTALE}

\begin{tabular}{c}
25 \\
\hline 26 \\
8 \\
17 \\
11 \\
2 \\
1 \\
9 \\
5 \\
1 \\
\hline 1 \\
2 \\
\hline 2
\end{tabular}

\begin{tabular}{|c|c|c|c|c|c|c|}
\hline GRUPPO & SIEROTIPO & AMBI & ALIM & UOMO & C. $\mathbf{Q}$. & TOTALE \\
\hline \multirow[t]{5}{*}{ D O:9 } & Enteritidis & 15 & 2 & 84 & 9 & 110 \\
\hline & Israel & 1 & & & & 1 \\
\hline & Napoli & 2 & & 2 & & 4 \\
\hline & Panama & 23 & & & & 23 \\
\hline & Kapemba & & & $\mathrm{I}$ & & 1 \\
\hline \multirow[t]{8}{*}{ EO:3, I 0, I 5} & Anatum & 35 & 1 & & & 36 \\
\hline & London & 47 & 2 & 4 & & 53 \\
\hline & Newlands & 2 & & & & 2 \\
\hline & Give & 8 & & 2 & & 10 \\
\hline & Muenster & 2 & & & & 2 \\
\hline & Nchanga & 3 & & & & 3 \\
\hline & Amsterdam & 2 & & & & 2 \\
\hline & Sinstorf & 2 & & 1 & & 3 \\
\hline \multirow[t]{2}{*}{ F O:I I } & Chingola & 1 & & & & 1 \\
\hline & Veneziana & 138 & & 2 & & 138 \\
\hline \multirow[t]{2}{*}{ G O:13 } & Kedougou & 1 & & & & 1 \\
\hline & Demerara & $\mathrm{I}$ & & & & 1 \\
\hline \multirow[t]{2}{*}{$10: 16$} & Salford & & & & 3 & 3 \\
\hline & Gaminara & $\mathrm{I}$ & & & & $\mathrm{I}$ \\
\hline К O: I 8 & Cerro & 2 & & & & 2 \\
\hline \multirow[t]{4}{*}{ G2 O:23 } & Demerara & 3 & & & & 3 \\
\hline & Putten & & & 1 & & $\mathrm{I}$ \\
\hline & Kedougou & $\mathrm{I}$ & & & & $\mathrm{I}$ \\
\hline & Poona & 1 & & 1 & 1 & 3 \\
\hline \multirow[t]{2}{*}{ H O:6.14 } & Caracas & & & & & \\
\hline & Horsham & 8 & & & & 8 \\
\hline \multirow[t]{3}{*}{ M O:28 } & Umbilo & 7 & & & & 7 \\
\hline & Pomona & 2 & & & & 2 \\
\hline & Arizonae & $\mathrm{I}$ & & & & 1 \\
\hline N O:30 & Arizonae & $\mathrm{I}$ & & & & $\mathrm{I}$ \\
\hline O 0:35 & Arizonae & 2 & & & & 2 \\
\hline P O:38 & Arizonae & 4 & & & & 4 \\
\hline Q O:39 & Arizonae & 1 & & & & 1 \\
\hline R O:40 & Arizonae & 21 & & & & 21 \\
\hline S 0:4I & Arizonae & 1 & & & & 1 \\
\hline T O:42 & Brive & 1 & & & & 1 \\
\hline \multirow[t]{2}{*}{ U O:43 } & Arizonae & 12 & 1 & & & 13 \\
\hline & Ahuza & 1 & & & & $\mathrm{I}$ \\
\hline V O:44 & Malika & 1 & & & & 1 \\
\hline X 0:47 & Arizonae & 2 & & & & 2 \\
\hline Z 0.50 & Arizonae & 38 & & & & 38 \\
\hline TOTALE & & 900 & 28 & 364 & 30 & 1322 \\
\hline
\end{tabular}

AMB ambientali, ALIM alimentari, UOMO umani, C.Q. controllo qualità 
Tabella 4. Anno 2005: raffronto fra i primi 10 sierotipi isolati da matrici umane e ambientali

\begin{tabular}{lccc}
\hline & AMBIENTE & & UOMO \\
\hline Sierotipo & $\mathbf{N}^{\circ}$ isolamenti & Sierotipo & $\mathbf{N}^{\circ}$ isolamenti \\
\hline Typhimurium & 15 I & Typhimurium & 205 \\
\hline Veneziana & 138 & Enteritidis & 84 \\
\hline Infantis & 56 & Infantis & 24 \\
\hline London & 47 & Muenchen & 7 \\
\hline Derby & 38 & Manhattan & 7 \\
\hline Agona & 38 & Newport & 6 \\
\hline Arizonae 50 & 38 & Montevideo & 4 \\
\hline Anatum & 35 & Braenderup & 4 \\
\hline Montevideo & 34 & London & 4 \\
\hline Nitra & 25 & Livingstone & 3 \\
\hline
\end{tabular}

\section{ELENCO REFERENTI}

D.sa Bacchi Marta ARPA Forlì V.le Salinatore N. 20 - 47100

D.sa Benini FrancaASL Ravenna Osp.S.M. delle Croci V.le Randi n5 - 48100.

D.sa Bernardi Daniela ARPA Piacenza Via XXI Aprile nº 48 - 29100

Dr. Bevilacqua Loris S.I.P. ASL Ravenna Largo Chartres n 3 - 48100

D.sa Camellini Loretta ARPA Reggio Emilia Via Amendola n² - 42100

Dr. Cipolloni Paolo ASL Cesena Ospedale ‘Bufalini’ V.le Ghirotti n’286 - 47023

Sigg.Crea e/o Paoli.S.I.P. Castelnuovo Monti Via Boschi n ${ }^{\circ}$ - 42035

Dr. David Lev ARPA Rimini Via Gambalunga n 83 - 47037

D.sa Del Greco Daniela ARPA Parma Via Spalato ${ }^{\circ} 4$ - 43100

D.sa Fiumana Emanuela ASL Forlì Via della Rocca n 12 - 47100

D.sa Giacobbi Francesca ARPA Bologna Via Triachini n ${ }^{\circ} 5$ - 40100

Sig.na Montanari Nadia S.I.P. Amb. 107 Reggio Emilia Via Amendola n² - 42100

Dr. Montini Giuseppe ASL Forlì Ospedale Pierantoni Via C. Forlanini - 47100

D.sa Natali Patrizia ARPA Modena V.le Fontanelli n ${ }^{\circ} 21$ - 41100

D.sa Pagnani Monica ARPA Ravenna Via Alberoni 17/19 - 48100

Dr. Pazzi Lorenzo Tecnolab Alimentare Forlì Via S. Pellico n²1 - 47100

Responsabile ASL Correggio (RE) P.zza Recordati n ${ }^{\circ} 8$ - 42015

Dr.sa Rossi M.Rita ASL Ferrara Osp. S. Anna C.so Giovecca n²03 - 44100

D.sa Scalcione Agata Centro “J. Fleming” Faenza Via Case Nuove n 15 - 48018

D.sa Tampieri M.Luisa ARPA Ferrara Corso Giovecca n ${ }^{\circ} 169$ - 44100

Dr.sa Testa Giovanna ASL Rimini Osp. 'Infermi' Via Settembrini n’2 - 47900

Assistenti sanitarie S.I.P. Guastalla (RE) P.zza Matteotti nº - 42016.

\section{BIBLIOGRAFIA}

1. Bacchi M, Cirillo G, Ranieri M. 'Enter-net, la rete di sorveglianza delle infezioni da Salmonella e coliformi' ARPA-Rivista 2004 Anno VII n5 p.34-5.

2. Bergey's Manual of Systematic Bacteriology vol $\mathrm{I}^{\circ}$ 1986; 427-58.

3. Caprioli A, Luzzi I, et al. ISTISAN Congressi 03/C5 2003; 1-66.

4. Centro di Riferimento Regionale Lazio Enterobatteri Patogeni "Rapporto regionale sulla sorveglianza di Laboratorio: anni 1997-2003" Roma 2004

5. Caprioli A, Luzzi I, et al. ISTISAN Congressi 04/C4 2004; 1-107

6. Caprioli A, Luzzi I, et al. ISTISAN Congressi 05/C12 2005; 1-81

7. Cirillo G. Evoluzione epidemiologica del genus Salmonella: 25 anni di osservazioni sul territorio di Forlì. Atti RER e Prov.di Ferrara 1986; 63-6.

8. Cirillo G. La sorveglianza delle salmonellosi legate all'ambiente. Atti ISS Roma 2005; 29-30.

9. Cirillo G. Sistema di sorveglianza Enternet: isolamenti di salmonella da fonti ambientali. Atti ISS Roma 2003; 6-7.
10. Cirillo G. Il ciclo biologico nelle patologie alimentari. Atti AMCLI Padova 2004; 84-6.

11. Edward PR, Edwing WH. Identification of Enterobacteriacee. II edit. 1962; 93-172.

12. Graziani C, Galletta P, et al. Rapporto ISTISAN 05/27: Infezioni da Salmonella: diagnostica, epidemiologia e sorveglianza. Roma 2005.

13. Popoff MY\& Le Minor L. Antigenic Formulas of the Salmonella serovars. Istitut Pasteur 1997.

14. Tozzi AE, et al. Il Sistema di Sorveglianza ENTERNET: isolamenti di Salmonella dall'uomo. Atti ISS 2003; 3-4.

15. Zavanella M. Tipizzare le Salmonella. Fondazione iniziative zooprofilattiche e zootecniche. Brescia 2001

\section{Giuseppe Cirillo}

Eccellenza Biologia e Microbiologia S\&R ARPA Forlì-Cesena

V.le Salinatore 20 - 47100 Forlì

Tel 0543451428 - Fax 0543451451

E-mail: gcirillo@fo.arpa.emr.it 